\title{
An Alternative Method for the Dissolution of Enrofloxacin Tablets
}

\author{
G. R. Foresti ${ }^{1,2}$, N. Becker ${ }^{1,2}$, A. S. Silva ${ }^{1,2}$, W. R. R. Almeida' ${ }^{1}$ M. D. Malesuik ${ }^{1,2}$, \\ S. E. Hass ${ }^{2,}$ and F. E. B. Silva ${ }^{1,2, *}$ \\ ${ }^{1}$ Núcleo de Pesquisa em Fármacos e Medicamentos, UNIPAMPA, Campus Uruguaiana, RS, Brasil \\ ${ }^{2}$ Programa de Pós-graduação em Ciências Farmacêuticas (PPGCF)-UNIPAMPA, Campus Uruguaiana, RS, Brasil
}

\begin{abstract}
Enrofloxacin is a fluoroquinolone for veterinary use; it has low aqueous solubility and relatively high permeability. Dissolution may be the limiting step in absorption for solid dosage forms having these characteristics. Considering this, in vitro dissolution tests are indicated to evaluate batch-to-batch quality and to support pharmaceutical equivalence studies. In this study, an alternative dissolution profile was developed for tablets containing enrofloxacin. The selected method uses a $0.01 \mathrm{~N} \mathrm{HCl}$ medium, paddle apparatus, and 50-rpm speed. The samples were analyzed by UV spectroscopy at 273 $\mathrm{nm}$. The results confirm that the proposed method is suitable for routine quality control of enrofloxacin tablets and the comparison of the dissolution profiles of different commercial formulations.
\end{abstract}

KEYWORDS: Enrofloxacin; dissolution; tablets.

\section{INTRODUCTION}

D issolution tsting of active pharmaceuticals in solid dosage forms (tablets and capsules) is a crucial factor to certify formulation quality and homogeneity $(1,2)$. Analytical methodologies to evaluate the dissolution of human medicinal products and to compare dissolution profiles to attest pharmaceutical equivalence are described in official pharmaceutical compendia and regulated by the Brazilian Agency for Sanitary Vigilance (ANVISA) (3). However, there are no official codes or regulations describing how dissolution profiles for veterinary drugs should be performed.

Recently, the USP published two articles $(2,4)$ that stimulated discussion on solubility criteria and concepts about in vitro dissolution and the Biopharmaceutics Classification System (BCS) for veterinary medicinal products. According to the authors, considering the principles of Good Manufacturing Practices (GMPs), the inclusion of BCS concepts would be extremely beneficial for veterinary medicine.

Another factor to be considered in veterinary pharmaceuticals dissolution refers to anatomical and physiological differences among species, which can modify in vivo and in vitro bioavailability (5-7). In this context, it is important to develop analytical methods considering the specific characteristics from each formulation and physiological characteristics of individual species.

Enrofloxacin is a fluoroquinolone antibiotic used in veterinary practice to treat respiratory and gastrointestinal infections (8). This drug is available as a tablet, an injectable, and an oral solution. However, there are few reports on quality control methods for these formulations, and there are no data on dissolution profiles for tablets. Because of

*Corresponding author. the low solubility of enrofloxacin, dissolution may be the rate-limiting step to dosage form absorption; therefore, it becomes necessary to evaluate the drug dissolution profile. This study aims to develop analytical methodology to evaluate dissolution profiles of enrofloxacin tablets and compare the profiles of different formulations available commercially.

\section{MATERIALS AND METHODS Materials}

Enrofloxacin (1-cyclopropyl-7-(4-ethyl-1-piperazinyl)6-fluoro-1,4-dihydro-4-oxo-3-quinolinecarboxylic acid) reference standard (99.7\%) was kindly supplied by VetPharma (Brazil). This raw material was analyzed according to the USP monograph (9). Tablets containing 50 $\mathrm{mg}$ of enrofloxacin were obtained commercially. All other reagents used were analytical grade.

\section{Samples}

In Brazil, the reference product for veterinary use is defined as an "innovative product registered and marketed in a federal jurisdiction whose efficacy, safety, and quality has been scientifically proven at registration" (10). Thus, drug product inclusion in the Reference Drugs List qualifies it as a comparator in pharmaceutical equivalence tests and in bioequivalence studies for generic drug registrations.

Currently, the Ministry of Agriculture (MAPA) does not provide listings containing reference veterinary drugs, despite legislation that allows generic drug registration for veterinary use in Brazil. For this reason, the drug product considered as a reference by FDA has been selected for comparison with five commercially available enrofloxacin tablets in this study. 


\section{Drug Solubility and Sink Conditions}

Drug solubility was tested in different solvents to find a medium that ensures sink conditions. The following media were evaluated: $0.1 \mathrm{~N} \mathrm{HCl}, 0.01 \mathrm{~N} \mathrm{HCl}, \mathrm{pH} 4.5$ acetate buffer, pH 5.8 phosphate buffer, $\mathrm{pH} 6.4$ phosphate buffer, and $\mathrm{pH}$ 7.8 phosphate buffer. Enrofloxacin samples $(166.6 \mathrm{mg} / \mathrm{mL}$ ) were sonicated at $37 \pm 0.5^{\circ} \mathrm{C}$ for $15 \mathrm{~min}$ in different media. After filtration, the samples were diluted and analyzed by UV spectroscopy at $273 \mathrm{~nm}$. The solubility in each medium was determined in triplicate.

\section{Dissolution Test Development}

The dissolution method was developed using a Varian model VK 7000 dissolution apparatus equipped with six vessels. The samples were analyzed in a Perkin Elmer UV spectrophotometer (Lambda 35). All dissolution media were first deaerated in an ultrasonic bath (Unique model 2850A USC) for $10 \mathrm{~min}$. Different experimental conditions were tested as shown in Table 1. Sample stability in dissolution media was evaluated by UV drug quantitation after $24 \mathrm{~h}$ storage at ambient temperature $\left(T_{\mathrm{amb}}\right)$.

Table 1. Experimental Conditions

\begin{tabular}{lc}
\hline Temperature & $37^{\circ} \mathrm{C}$ \\
\hline Apparatus & Paddle \\
\hline Velocity & $50 \mathrm{rpm}$ \\
\hline & $0.1 \mathrm{~N} \mathrm{HCl}$ \\
& $0.01 \mathrm{~N} \mathrm{HCl}$ \\
Dissolution media & $\mathrm{pH} 4.5$ acetate buffer \\
& $\mathrm{pH} 6.8$ phosphate buffer \\
& $\mathrm{pH} 7.4$ phosphate buffer \\
\hline Volume & $900 \mathrm{~mL}$ \\
\hline Sampling time (min) & $5,10,15,20,30,40,50,60$ \\
\hline UV Detection & $273 \mathrm{~nm}$ \\
\hline
\end{tabular}

According to the Veterinary International Conference on Harmonisation $(\mathrm{VICH})$, validation of dissolution methods in veterinary products must include parameters of specificity, linearity, precision, and accuracy $(11,12)$. Considering these guidelines, the above-mentioned parameters were evaluated along with robustness. To determine the specificity, a placebo formulation was used. The excipients of the placebo formulation were calculated from the average weight of the reference sample and the concentration used in immediate-release tablets (13). A flavor excipient was added to the placebo formulation. The method is considered specific, with interference not exceeding $2 \%$ of drug absorbance at $273 \mathrm{~nm}$. The linearity results were subjected to a statistical analysis of variance (ANOVA). The results for repeatability and intraday precision are considered suitable with an RSD less than $2 \%$. The method is accurate for testing recoveries obtained at 95-105\%. The robustness test evaluated drug dissolution in aerated and deaerated media. The method is considered robust for dissolution values without significant difference (14).

To analyze the in vitro release kinetics, zero-order, first-order, Higuchi, and Hixson-Crowell mathematical models were used. The mathematical model that adequately expressed the enrofloxacin dissolution profile was selected according to the correlation coefficient $(r)$. The time corresponding to $80 \%$ dissolution $\left(t_{80}\right)$ was used to characterize the drug release matrix and infer a $Q$ value. This $Q$ value corresponds to the minimum percentage dissolution at a specified time and can be used as an acceptance parameter in routine dissolution testing.

\section{Dissolution Profiles Comparison}

The dissolution profiles of five formulations from different manufacturers were compared, and the similarity factor $f_{2}$ was then determined (3).

The parameter dissolution efficiency $(D E)$ was used to characterize the drug release profile of the dosage form $(15,16)$. $D E$ is the area under the curve within a time range and is expressed as a percentage (17).

\section{RESULTS AND DISCUSSION \\ Solubility Determination and Sink Conditions}

Enrofloxacin is an amphoteric drug with a $\mathrm{p} K_{\mathrm{a} 1}=5.94$ corresponding to the carboxyl group and $\mathrm{p} K_{\mathrm{a} 2}=8.70$ corresponding to the piperazinyl group (isoelectric point at $\mathrm{pH}=7.32$ ). The octanol-aqueous buffer $\mathrm{pH} 7.0$ partition coefficient for enrofloxacin is 3.48. Therefore, this drug is considered highly lipophilic (18). According to Seedher (19), enrofloxacin water solubility is $146 \mathrm{mg} / \mathrm{mL}$, and it is listed as poorly soluble based on pharmacopeial criteria. Enrofloxacin has a chemical structure similar to that of ciprofloxacin (its major metabolite), differing in piperazinyl group acetylation, which provides higher lipophilicity. Because of the characteristics described above, it can be inferred that enrofloxacin presents more difficult dissolution in aqueous solvents and gastrointestinal fluids than ciprofloxacin. According to the USP, in dissolution and permeability studies with human pharmaceuticals, the solubility determination should be based on the highest dose (in $\mathrm{mg}$ ) approved for use. However, for veterinary pharmaceuticals, the dose is administered based on body weight. For this reason, the amount $(\mathrm{mg})$ per animal weight ( $\mathrm{kg})$ per day and gastric volume (2) should be considered in solubility determinations for veterinary pharmaceuticals. Therefore, USP (2) proposed to classify veterinary pharmaceutical solubility based on the estimated $D_{0}$ (the dose number) according to the following equation:

$$
D_{0}=(M / V) / C
$$

where $D_{0}$ is dose number, $M$ is dose strength of the tablet or capsule, $V$ is volume administered $(35 \mathrm{~mL}$ based upon the body weight of beagle dogs, $10-11 \mathrm{~kg}$ ), and $C$ is drug 
solubility $(\mathrm{mg} / \mathrm{mL})$.

A $D_{0} \geq 1$ has been used as a definition of a lowsolubility drug, whereas a $D_{0}<1$ defines a highly soluble drug. Enrofloxacin showed a $D_{0}=9.78$ and was classified as low solubility. Considering the BCS, enrofloxacin is classified as Class 2 (low solubility/high permeability). For any classification, dissolution is the rate-limiting step of absorption for enrofloxacin, and modifications in the formulation or manufacturing processes could alter drug bioavailability. Table 2 shows the results obtained from solubility tests and sink conditions. According to the results, distilled water and $\mathrm{pH} 5.8$ phosphate buffer media did not reach sink conditions (Table 2). This result agrees with the results obtained by Seedher and Agarval (19), which established drug solubility in aqueous media at $146 \mathrm{mg} /$ $\mathrm{mL}$. According to Lizondo et al. (18), enrofloxacin exhibits a low solubility at a pH of about 5.5 .

Table 2. Solubility Test Results

\begin{tabular}{lc}
\hline \multicolumn{1}{c}{ Medium } & \% solubilized \pm RSD $(\mathbf{n}=\mathbf{3})$ \\
\hline $0.1 \mathrm{~N} \mathrm{HCl}$ & $99.9 \pm 0.9$ \\
$0.01 \mathrm{~N} \mathrm{HCl}$ & $100.0 \pm 0.7$ \\
Acetate buffer pH 4.5 & $100.7 \pm 0.3$ \\
Phosphate buffer pH 7.4 & $100.7 \pm 0.5$ \\
Phosphate buffer pH 6.8 & $98.2 \pm 0.8$ \\
Phosphate buffer pH 5.8 & $39.1 \pm 14.3$ \\
Distilled water & $72.6 \pm 3.5$ \\
\hline
\end{tabular}

\section{Dissolution Test Development}

The dissolution test systems commercially available simulate human physiological conditions and consist of a vessel containing dissolution medium and an apparatus that moves this medium (simulating peristalsis). Since there are no specific equipment or apparatus for veterinary pharmaceuticals dissolution, equipment for human pharmaceuticals has been used during this study. Therefore, method development was based on enrofloxacin solubility and dissolution medium selection.

Dissolution medium selection is an important factor in drug dissolution and should be based on drug solubility in its relationship to dose. Accordingly, it is necessary to define sink conditions for dissolution medium tested. Sink conditions can be achieved when the medium volume used is three times higher than drug saturation concentration. This ensures that dissolution rate is not diminished by approaching saturation concentration (20).

During dissolution test development, the reference drug dissolution profile was evaluated in different media. Profiles obtained for proposed conditions are shown in Figure 1. Data are expressed as percentage dissolved versus time.

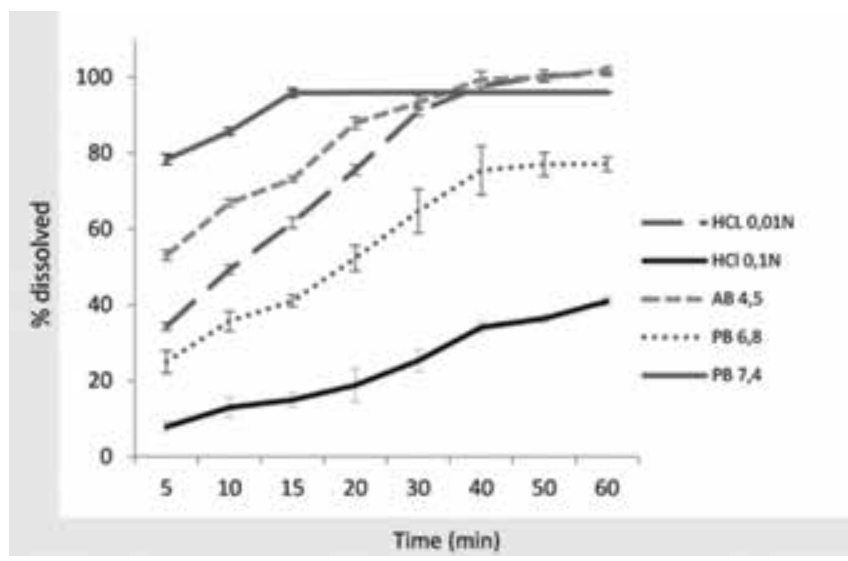

Figure 1. Dissolution profiles in (PB 7.4) $\mathrm{pH} 7.4$ phosphate buffer, $(P B$ 6.8) $\mathrm{pH}$ 6.8 phosphate buffer, (AB 4.5) pH 4.5 acetate buffer, $0.1 \mathrm{~N} \mathrm{HCl}$, and $0.01 \mathrm{~N}$ $\mathrm{HCl}$.

According to ANVISA, dissolution media are considered discriminating if drug dissolution is below $85 \%$ in $15 \mathrm{~min}$ (3). This condition was achieved for all media except for PB 7.4, although PB 6.8 and $0.1 \mathrm{~N} \mathrm{HCl}$ media did not release drug completely from matrix excipients in a specified time. Thus, only $0.01 \mathrm{~N} \mathrm{HCl}$ and $\mathrm{AB} 4.5$ media were adequate to evaluate enrofloxacin dissolution. The $0.01 \mathrm{~N}$ $\mathrm{HCl}$ dissolution medium was selected for further studies.

After the experimental conditions were selected, the dissolution test was validated for specificity, linearity, precision, accuracy, and robustness. The results are within the criteria established by ICH for analytical methodologies validation (14).

As described in Table 3, a study of drug release kinetics was performed using mathematical models. The respective determination coefficients $\left(r^{2}\right)$ were obtained through dissolution linearization profiles. The kinetics model considered most appropriate (higher determination coefficient) was the Higuchi model. The Higuchi model was used to calculate dissolution time $\geq 80 \% \quad\left(t_{80 \%}\right)$. The parameter $t_{80 \%}$ was $24.83 \mathrm{~min}$, and suggests a 30 min dissolution time and a $Q$ value equal to 80 for this pharmaceutical dosage form.

Table 3. Reaction Order and Correlation Coefficients

\begin{tabular}{lcc}
\hline \multicolumn{1}{c}{ Model } & \multicolumn{1}{c}{ Linear fit } & (r2) \\
\hline Zero order & \% dissolved $=26.012+2.274 \mathrm{t}$ & 0.9796 \\
First order & In \% dissolved $=3.589+0.028 \mathrm{t}$ & 0.8657 \\
Higuchi & \% dissolved $=-0.232+16.101 \mathrm{t} 0.5$ & 0.9937 \\
Hixson-Crowell & \% dissolved1/3 $=1.354-0.037 \mathrm{t}$ & 0.8932 \\
\hline
\end{tabular}

\section{Dissolution Profile Comparison}

Four brands of enrofloxacin tablets were tested for dissolution profiles and compared with the reference drug. The dissolution profiles of samples from different manufacturers (Am1, Am2, Am3, and Am4) and the 
reference drug (Reference) are shown in Figure 2. The $f_{2}$ values were calculated, and the comparative results are shown in Table 4.

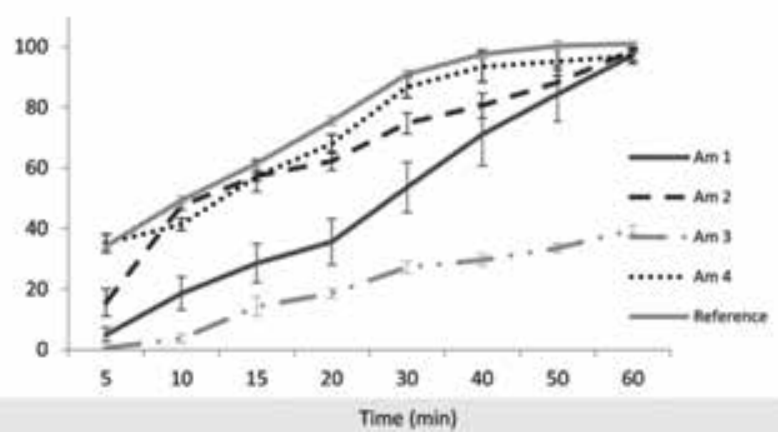

Figure 2. Dissolution profiles of tested formulations.

Table 4. $f_{2}$ Values of Tested Formulations

\begin{tabular}{cc}
\hline Formulation & $f_{2}$ \\
\hline Reference $\times$ Am1 & 55.25 \\
Reference $\times$ Am2 & 69.61 \\
Reference $\times$ Am3 & 42.41 \\
Reference $\times$ Am4 & 81.53 \\
\hline
\end{tabular}

The formulations Am1, Am2, and Am4 show dissolution profiles similar to the reference drug according to the criteria established by ANVISA, which requires an $f_{2}$ value between 50 and 100 (3). However, the largest $f_{2}$ was obtained for sample Am4, which contained artificial flavor excipient like the reference formulation.

Dissolution kinetics of sample Am4 is described by the same model as the reference drug (Higuchi model), and sample Am3 represents the greatest distance from the proposed model showing low correlation (Table 5). The Higuchi model is characteristic of controlled-release by a diffusion process and is based on Fick's Law. This model is frequently used to describe controlled release from a matrix system (21). Table 5 shows $D E$ values and $D E$ differences between reference drug and the remaining samples. According to Anderson et al. (22), dissolution profiles with variations in $D E$ exceeding $10 \%$ had $f_{2}$ values less than 40 . However, the difference in $D E$ values is not significant enough if the $f_{2}$ value is between 40 and 50 . For this reason, a decision based on the $f_{2}$ parameter would not be supported by results obtained for $D E$. This occurs because the calculation of $f_{2}$ parameter does not consider the data dispersion (23). According to this study, sample Am1 would be included in this situation, as it has been considered similar based on the parameter $f_{2}$. On the other hand, sample Am1 presented differences in $D E$ values greater than $10 \%$ compared with the reference sample.
Table 5. Parameters of Linearization and Dissolution Efficiency

\begin{tabular}{ccccc}
\hline Formulation & $\begin{array}{c}r^{2} \\
\text { (Higuchi Model) }\end{array}$ & t80 (min) & DE (\%) & $\Delta \mathrm{DE}$ \\
\hline Reference & 0.9937 & 24.83 & 76.8 & - \\
Am1 & 0.8990 & 63.63 & 51.7 & -25.1 \\
Am2 & 0.9493 & 35.41 & 75.21 & 1.59 \\
Am3 & 0.8525 & 255.04 & 22.21 & -54.9 \\
Am4 & 0.9888 & 28.31 & 73.57 & -3.23 \\
\hline
\end{tabular}

\section{CONCLUSIONS}

The dissolution test developed and validated for enrofloxacin tablets is considered satisfactory. The most discriminating conditions for the dissolution testing of enrofloxacin tablets (i.e., $0.01 \mathrm{~N} \mathrm{HCl}$, paddle apparatus, stirring speed of $50 \mathrm{rpm}$, and sampling time of $30 \mathrm{~min}$ ) appear to be optimal. The validation shows that the dissolution test is appropriate for quantification of enrofloxacin in tablet pharmaceutical form for in vitro studies. The method is adequate for use in quality control testing of enrofloxacin tablets since a dissolution test is not indicated in an official monograph. The proposed method is simple, relatively inexpensive, easy to perform, and has the practicality required for routine laboratory quality control to ensure the safety and therapeutic efficacy of enrofloxacin tablets. Thus, it can be used in collaborative studies of the pharmaceutical equivalence in veterinary medicines.

\section{REFERENCES}

1. Storpirtis, S.; Gai, M. N.; Chiann, C.; Gonçalves, J. E. Biofarmacotécnica, 1st ed.; Guanabara Koogan: Rio de Janeiro, 2009.

2. Apley, M.; Crist, B.; Gonzalez, M. A.; Hunter, R. P.; Martinez, M. N.; Modric, S.; Papich, M. G.; Parr, A. F.; Riviere, J. E.; Marques, M. R. C. Stimuli to the Revision Process: Solubility Criteria for Veterinary Drugs. Pharm. Forum 2012, 38 (4), 1-12.

3. Realização dos Estudos de Equivalência Farmacêutica e de Perfil de Dissolução Comparativo. RE no 31, Agência Nacional de Vigilância Sanitária, Ministério da Saúde: Brasil, 2010.

4. Martinez, M. N.; Papich, M. G.; Riviere J. E. Stimuli to the Revision Process: Veterinary Application of In Vitro Dissolution Data and the Biopharmaceutics Classification System. Pharm. Forum 2013, 30 (6), 1-20.

5. Martinez, M.; Amidon, G.; Clarke, L.; Jones, W. W.; Mitra, A.; Riviere, J. Applying the biopharmaceutics classification system to veterinary pharmaceutical products: Part II: Physiological considerations. Adv. Drug Deliv. Rev. 2002, 54 (6), 825-850. DOI: 10.1016/ S0169-409X(02)00071-6.

6. Martinez, M.; Augsburger, L.; Johnston, T.; Jones, W. W. Applying the Biopharmaceutics Classification System to veterinary pharmaceutical products: Part I: Biopharmaceutics and formulation considerations. Adv. 
Drug Deliv. Rev. 2002, 54 (6), 805-824. DOI: 10.1016/ S0169-409X(02)00070-4.

7. Sutton, S.C.Companion animal physiology and dosage form performance. Adv. Drug Deliv. Rev. 2004, 56 (10), 1383-1398. DOI: 10.1016/j.addr.2004.02.013.

8. Martinez, M.; McDermott, P.; Walker, R. Pharmacology of the fluoroquinolones: A perspective for the use in domestic animals. Vet. J. 2006, 172 (1), 10-28. DOI: $10.1016 /$ j.tvjl.2005.07.010.

9. The United States Pharmacopeia and National Formulary USP 35-NF 30; The United States Pharmacopeial Convention, Inc.: Rockville, MD, 2012.

10. Medicamento Genérico de Uso Veterinário. Lei no 12.689, Presidência da República: Brasil, 2012.

11. International Cooperation on Harmonisation of Technical Requirements for Registration of Veterinary Medicinal Products (VICH). GL 1-Validation of Analytical Procedures: Definition and Terminology; International Federation for Animal Health: Brussels, 1998.

12. International Cooperation on Harmonisation of Technical Requirements for Registration of Veterinary Medicinal Products (VICH). GL 39-Test procedures and acceptance criteria for new veterinary drug substances and new medicinal products: chemical substances; International Federation for Animal Health: Brussels, 2005.

13. Kibbe, A. H., Ed. Handbook of Pharmaceutical Excipients, 3rd ed.; American Pharmaceutical Association: Washington, DC, 2000.

14. International Conference on Harmonisation ofTechnical Requirements for Registration of Pharmaceuticals for Human Use. Validation of Analytical Procedures: Text and Methodology, Q2(R1); ICH Harmonised Tripartite Guideline: Geneva, Switzerland, 2005.

15. Serra, C. H. R.; Storpirtis, S. Comparação de perfis de dissolução da cefalexina através de estudos de cinética e eficiência de dissolução (ED\%). Braz. J. Pharm. Sci. 2007, 43 (1), 79-88.

16. Silva, A. P. C.; Meneghini, L. Z.; Bajerski, L.; Carini, J. P.;
Fialho, S. L.; Mayorga, P.; Fröelich, P. E. Discriminatory Dissolution Test for Tablets Containing $a-$ and $\beta$-Thalidomide Polymorphs. Dissolution Technol. 2013, 20 (1), 19-25.

17. Khan, K. A. The concept of dissolution efficiency. J. Pharm. Pharmacol. 1975, 27 (1), 48-49. DOI: 10.1111/ j.2042-7158.1975.tb09378.x.

18. Lizondo, M.; Pons, M.; Gallardo, M.; Estelrich, J. Physicochemical properties of enrofloxacin. J. Pharm. Biomed. Anal. 1997, 15 (12), 1845-1849. DOI: 10.1016/ S0731-7085(96)02033-X.

19. Seedher, N.; Agarwal, P. Various solvent systems for solubility enhancement of enrofloxacin. Indian J. Pharm. Sci. 2009, 71 (1), 82-87. DOI: 10.4103/0250474X.51958.

20. Phillips, D. J.; Pygall, S. R.; Cooper, V. B.; Mann, J. C. Overcoming sink limitations in dissolution testing: a review of traditional methods and the potential utility of biphasic systems. J. Pharm. Pharmacol. 2012, 64 (11), 1549-1559. DOI: 10.1016/S0731-7085(98)00011-9.

21. Dash, S.; Murthy, P. N.; Nath, L.; Chowdhury, P. Kinetic modeling on drug release from controlled drug delivery systems. Acta Pol. Pharm. 2010, 67 (3), 217223.

22. Anderson, N. H.; Bauer, M.; Boussac, N.; Khan-Malek, R.; Munden, P.; Sardaro, M. An evaluation of fit factors and dissolution efficiency for the comparison of in vitro dissolution profiles. J. Pharm. Biomed. Anal. 1998, 17 (4-5), 811-822. DOI: 10.1016/S0731-7085(98)00011-9.

23. Costa, P. An alternative method to the evaluation of similarity factor in dissolution testing. Int. J. Pharm. 2001, 220 (1-2), 77-83. DOI: 10.1016/S03785173(01)00651-2. 